\title{
ON VARIATION FORMULAS FOR UNIVALENT FUNCTIONS
}

\author{
BY \\ A. W. GOODMAN
}

1. Introduction. Let $U$ denote the class of functions

$$
f(z)=z+\sum_{k=2}^{\infty} a_{k} z^{k}
$$

which are regular and univalent in $|z|<1$, that is $f(z)$ takes no value more than once in the open unit circle. It has been conjectured that if $f(z) \in U$, then $\left|a_{n}\right| \leqq n$, and an extensive literature has appeared, directed toward proving this conjecture in various special cases. Perhaps the most penetrating result presently known is the Schiffer, Schaeffer, Spencer $[2 ; 3]$ differential equation for an extremal function. Their result, stated precisely, is as follows.

Theorem 1. Let $n \geqq 2$ be a fixed integer and let

$$
F(z)=z+\sum_{k=2}^{\infty} A_{k} z^{k}, \quad F(z) \in U,
$$

be such that $A_{n}>0$, and $f(z) \in U$ implies that $\left|a_{n}\right| \leqq A_{n}$. Then $F(z)$ satisfies the differential equation

$$
\sum_{k=2}^{n} \frac{A_{n}^{(k)}}{F(z)^{k+1}}=\frac{1}{z^{2} F^{\prime}(z)^{2}}\left\{(n-1) A_{n}+\sum_{k=1}^{n-1} k\left(\bar{A}_{k} z^{n-k}+\frac{A_{k}}{z^{n-k}}\right)\right\}
$$

where $A_{n}^{(\mathbf{k})}$ is defined by the equation

$$
F(z)^{k}=\sum_{j=k}^{\infty} A_{j}^{(k)} z^{j}, \quad k=2,3,4, \cdots
$$

In this paper, we give a new proof of the above theorem. Although our proof is at least as simple as the earlier proof's, the main interest of the result lies in the fact that a new and presumably different method of variation in the family of univalent functions, which a priori might be expected to yield a new condition on the extremal function, instead leads to the Schiffer, Schaeffer, Spencer differential equation, (1.3).

Our methods are elementary in the extreme. We need only a formula for the variation of a branch point proved in [1], and that paper required merely the Riemann mapping theorem in a very simple case, and the basic properties of the Green's function. The same method yields variation formulas for

Received by the editors November 10, 1956. 
bounded univalent functions, and for multivalent functions.

2. Proof of the main theorem. Our method is this. Let $F(z)$ be an extremal univalent function as described in the theorem and form a second degree polynomial in $u=F(z)$,

$$
g(u)=u-u^{2} / 2 C_{1}
$$

which will be 2 -valent for $|z|<1$, with a critical point at $z=C$, where $F(C)$ $=C_{1}$, and a branch point at $B=g\left(C_{1}\right)=C_{1} / 2$. The branch point is then moved to $B^{*}=B+\lambda$, using the formula proved in [1, Equation (1.3)]. If $u=G^{*}(w)$ denotes the inverse function for the quadratic

$$
g^{*}(u)=u-\frac{u^{2}}{2 C_{1}+4 \lambda}
$$

where the branch point of $g^{*}(u)$ is $B^{*}$, then $G^{*}(w)$ applied to the varied 2valent function will give a new univalent function $F^{*}(z)$.

Let

$$
f(z)=g(F(z))=F(z)-F^{2}(z) / 2 C_{1}
$$

be the function 2-valent in $|z|<1$, with critical point at $z=C$, and branch point at $B$. Applying the Formula (1.3) from [1] with $f^{\prime \prime}(C)=-F^{\prime}(C)^{2} / F(C)$, one finds easily that for this 2-valent function

$$
\begin{aligned}
\delta f(z)=z F^{\prime}(z)(1 & \left.-\frac{F(z)}{C_{1}}\right)\left\{\frac{\lambda F(C) z}{\left(C F^{\prime}(C)\right)^{2}(C-z)}\right. \\
& \left.+\operatorname{con}\left(\frac{\lambda F(C)}{\left(C F^{\prime}(C)\right)^{2}}\right) \frac{\bar{C} z}{1-\bar{C} z}+\Re \frac{\lambda F(C)}{\left(C F^{\prime}(C)\right)^{2}}\right\}+O\left(\lambda^{2}\right),
\end{aligned}
$$

where con ( ) denotes the conjugate of the quantity enclosed by the parentheses. Although the inverse function $G^{*}(w)$ can be explicitly computed, it is better to proceed as though $g^{*}(u)$ were an arbitrary function and use Taylor series to approximate the inverse function. In this way we find that

$$
\begin{aligned}
F^{*}(z) & =G^{*}\left(f^{*}(z)\right)=G^{*}(f(z)+\delta f(z)) \\
& =G^{*}(f(z))+\delta f(z) G^{* \prime}(f(z))+O\left(\lambda^{2}\right) \\
& =G^{*}(g(u))+\delta f(z) G^{* \prime}(g(u))+O\left(\lambda^{2}\right) .
\end{aligned}
$$

If we write

$$
g^{*}(u)=g(u)+\epsilon
$$

where $\epsilon=\epsilon(u, \lambda)=O(\lambda)$, then it is easy to see that

$$
\begin{aligned}
G^{*}(g(u)) & =G^{*}\left(g^{*}(u)-\epsilon\right)=G^{*}\left(g^{*}(u)\right)-\epsilon G^{* \prime}\left(g^{*}(u)\right)+O\left(\lambda^{2}\right) \\
& =u-\epsilon / g^{\prime}(u)+O\left(\lambda^{2}\right) .
\end{aligned}
$$


Similarly $G^{* \prime}(g(u))=1 / g^{\prime}(u)+O(\lambda)$. If this result and Equation (2.7) are used in Equation (2.5) we obtain

$$
F^{*}(z)=u+\frac{\delta f(z)-\epsilon}{g^{\prime}(u)}+O\left(\lambda^{2}\right) .
$$

This is our basic equation. We now substitute in this equation $\delta f(z)$ as given by Equation (2.4), $g^{\prime}(u)$, from Equation (2.1) and

$$
\epsilon=g^{*}(u)=g(u)=u-\frac{u^{2}}{2 C_{1}+4 \lambda}-\left(u-\frac{u^{2}}{2 C_{1}}\right)=\frac{u^{2} \lambda}{C_{1}^{2}}+O\left(\lambda^{2}\right) .
$$

This gives the following result.

Lemma 1. Let $F(z) \in U$, and let $0<|C|<1$. Then

$$
\begin{aligned}
F^{*}(z)=F(z)-\lambda & \frac{F^{2}(z) / F^{2}(C)}{1-F(z) / F(C)}+z F^{\prime}(z)\left\{\frac{\lambda F(C) z}{\left(C F^{\prime}(C)\right)^{2}(C-z)}\right. \\
& \left.+\operatorname{con}\left(\frac{\lambda F(C)}{\left(C F^{\prime}(C)\right)^{2}}\right) \frac{\bar{C} z}{1-\bar{C} z}+\Re \frac{\lambda F(C)}{\left(C F^{\prime}(C)\right)^{2}}\right\}+O\left(\lambda^{2}\right)
\end{aligned}
$$

is again a univalent function, for all complex $\lambda$ sufficiently small.

Although it is not immediately obvious, Equation (2.10) is essentially the same as the Schiffer variation formula, Equation (29) of [3]. We will show that this is so in $\S 6$ where we consider the more general case of a multivalent function. For completeness we indicate how Equation (2.10) leads to Equation (1.3).

In Equation (2.10) $F^{* \prime}(0) \neq 1$, and hence in order to have a normalized univalent function, we take

$$
\begin{aligned}
F_{*}(z) & =\frac{F^{*}(z)}{F^{* \prime}(0)}=F^{*}(z)-F(z) \Re \frac{\lambda F(C)}{\left(C F^{\prime}(C)\right)^{2}}+O\left(\lambda^{2}\right) \\
& =z+\sum_{k=2}^{\infty} A_{k}^{*} z^{k} .
\end{aligned}
$$

Using the notation introduce by Equation (1.4), an easy computation shows that

$$
\begin{aligned}
A_{n}^{*}= & A_{n}-\lambda \sum_{k=2}^{n} \frac{A_{n}^{(k)}}{F(C)^{k}}+(n-1) A_{n} \Re \frac{\lambda F(C)}{\left(C F^{\prime}(C)\right)^{2}} \\
& +\sum_{k=1}^{n-1}\left\{k A_{k} \frac{\lambda F(C)}{\left(C F^{\prime}(C)\right)^{2}} \frac{1}{C^{n-k}}+k A_{k} \operatorname{con}\left(\frac{\lambda F(C)}{\left(C F^{\prime}(C)\right)^{2}}\right) \bar{C}^{n-k}\right\}+O\left(\lambda^{2}\right) .
\end{aligned}
$$


Now if $A_{n}>0$, and $A_{n}$ is maximal, then we must have $\Re\left(A_{n}^{*}-A_{n}\right) \leqq 0$ for all $\lambda$. The second term in the sum may be replaced by its conjugate, without altering the real part. Further $\lambda$ is an arbitrary complex number. Consequently for an extremal $F(z)$, we must have

$$
\begin{aligned}
0= & -\sum_{k=2}^{n} \frac{A_{n}^{(k)}}{(F(C))^{k}}+(n-1) A_{n} \frac{F(C)}{\left(C F^{\prime}(C)\right)^{2}} \\
& +\frac{F(C)}{\left(C F^{\prime}(C)\right)^{2}} \sum_{k=1}^{n-1} k\left(\frac{A_{k}}{C^{n-k}}+\bar{A}_{k} C^{n-k}\right) .
\end{aligned}
$$

Since $C, 0<|C|<1$ is an arbitrary complex number, $C$ may be replaced by $z$ in Equation (2.13). This gives Equation (1.3) and the proof of Theorem 1 is complete.

3. An extension of the method. In [1] a formula was given for the variation of a second order branch point. In this section we apply the same procedure used in $\$ 2$, to a suitable cubic polynomial in $F(z)$, and obtain a new differential equation for the extremal function $F(z)$. It turns out that the new differential equation can be deduced directly from the Schiffer Schaeffer Spencer one. Nevertheless, the author feels that it is desirable to record the main steps and to indicate the way in which the new equation can be derived from Equation (1.3).

This time, we take as our polynomial

$$
g(u)=u-\frac{u^{2}}{C_{1}}+\frac{u^{3}}{3 C_{1}^{2}}
$$

which has its critical point at $F(C)=C_{1}$, and branch point at $B=g\left(C_{1}\right)=C_{1} / 3$. Then when $B^{*}=B+\lambda$

$$
g^{*}(u)=u-\frac{u^{2}}{C_{1}+3 \lambda}+\frac{u^{3}}{3\left(C_{1}+3 \lambda\right)^{2}} .
$$

Again set $f(z)=g(F(z))$. Then Equation (2.27) of [1] applied to this $f(z)$ yields

$$
\begin{aligned}
\delta f(z)= & z F^{\prime}(z)\left(1-\frac{F(z)}{C_{1}}\right)^{2}\left\{J_{1}(C) \frac{3 C z-2 z^{2}}{(C-z)^{2}} \lambda\right. \\
& +J_{2}(C) \frac{z}{C-z} \lambda+\bar{J}_{1}(C) \frac{\bar{C} z-2 \bar{C}^{2} z^{2}}{(1-\bar{C} z)^{2}} \bar{\lambda}+\bar{J}_{2}(C) \frac{\bar{C} z}{1-\bar{C} z} \bar{\lambda} \\
& \left.+\Re\left(2 J_{1}(C) \lambda+J_{2}(C) \lambda\right)\right\}+O\left(\lambda^{2}\right),
\end{aligned}
$$

where 


$$
J_{1}(C)=\frac{F(C)^{2}}{\left(C F^{\prime}(C)\right)^{3}}, \quad J_{2}(C)=\frac{2 F^{\prime \prime}(C) F(C)^{2}}{C^{2} F^{\prime}(C)^{4}}
$$

Equations (3.1) and (3.2) yield

$$
\epsilon=g^{*}(u)-g(u)=\frac{3 C_{1} u^{2}-2 u^{3}}{C_{1}^{3}} \lambda+O\left(\lambda^{2}\right) .
$$

Using this together with Equations (3.4), (3.1) and the fact that $u=F(z)$, $C_{1}=F(C)$ in Equation (2.8) gives the new univalent function

$$
\begin{aligned}
F^{*}(z) & =F(z)-\lambda \frac{3 F(C) F(z)^{2}-2 F(z)^{3}}{F(C)^{3}(1-F(z) / F(C))^{2}} \\
& +z F^{\prime}(z)\left\{J_{1}(C) \frac{3 C z-2 z^{2}}{(C-z)^{2}} \lambda+J_{2}(C) \frac{z}{C-z} \lambda+\bar{J}_{1}(C) \frac{\bar{C} z-2 \bar{C}^{2} z^{2}}{(1-\bar{C} z)^{2}} \bar{\lambda}\right. \\
& \left.+\bar{J}_{2}(C) \frac{\bar{C} z}{1-\bar{C}_{z}} \bar{\lambda}+\Re\left(2 J_{1}(C) \lambda+J_{2}(C) \lambda\right)\right\}+O\left(\lambda^{2}\right) .
\end{aligned}
$$

This new univalent function is not normalized. If we form

$$
F_{*}(z)=F^{*}(z) / F^{* \prime}(0),
$$

find the $n$th coefficient, insert conjugates at appropriate places, and let $\lambda \rightarrow 0$, as in $\$ 2$, we arrive at the following result.

THEOREM 2. Under the conditions of Theorem 1, $F(z)$ satisfies the differential equation

$$
\begin{aligned}
\sum_{k=2}^{n} \frac{(k+1) A_{n}^{(k)}}{F(z)^{k}}= & (n-1) A_{n}\left(2 J_{1}(z)+J_{2}(z)\right) \\
& +\sum_{k=1}^{n-1} \frac{k A_{k}}{z^{n-k}}\left((n-k+2) J_{1}(z)+J_{2}(z)\right) \\
& +\sum_{k=1}^{n-1} k \bar{A}_{k} z^{n-k}\left(J_{2}(z)-(n-k-2) J_{1}(z)\right) .
\end{aligned}
$$

On the other hand if we differentiate Equation (1.3) with respect to $z$, and then multiply by $-F(z)^{2} / F^{\prime}(z)$, we also obtain Equation (3.11).

4. Bounded univalent functions. The method of $\$ 2$ can also be used to give a variational formula for bounded univalent functions. Let UB denote the class of functions

$$
F(z)=\sum_{n=1}^{\infty} A_{n} z^{n}, \quad A_{1}>0
$$


which are regular, univalent, and $|F(z)|<1$ for $|z|<1$. Let $C$ be a complex constant with $0<|C|<1$, and set $F(C)=C_{1}=b^{1 / 2} e^{i \beta}, b^{1 / 2}>0$. From $[1,(3.1)]$ the function

$$
g(u)=\frac{2 b^{1 / 2} e^{i \beta} u-(1+b) u^{2}}{(1+b) e^{i \beta}-2 b^{1 / 2} u}
$$

maps $|u|<1$ onto a doubly covered circle $|g|<1$ with a simple branch point at $g\left(C_{1}\right)=b e^{i \beta}$. Whence the function

$$
f(z)=g(F(z))
$$

maps $|z|<1$ onto a doubly covered region with a simple branch point at $b e^{i \beta}=f(C)$. We move this simple branch by an amount $\lambda$, both for $f(z)$ and $g(u)$. Let $f^{*}(z)$ and $g^{*}(u)$ be the functions mapping $|z|<1$ and $|u|<1$, respectively, onto these new Riemann surfaces, and let $G^{*}(w)$ be the inverse function of $g^{*}(u)$. Then obviously

$$
F^{*}(z)=G^{*}\left(f^{*}(z)\right)
$$

is again a function in the class UB. The computation of $F^{*}(z)$ now parallels that in $\S 2$, and Equation (2.8) is directly applicable. To compute the term $\epsilon / g^{\prime}(u)$, we observe that $\epsilon=g^{*}(u)-g(u)$ is given by $[1,(1.3)]$ applied to $g(u)$ with critical point at $u=C_{1}$. Computation gives

$$
g^{\prime}(u)=\frac{2(1+b)\left(b^{1 / 2} e^{2 i \beta}-(1+b) u e^{i \beta}+b^{1 / 2} u^{2}\right)}{\left((1+b) e^{i \beta}-2 b^{1 / 2} u\right)^{2}}
$$

and, since $g^{\prime}\left(C_{1}\right)=0$,

$$
\begin{aligned}
g^{\prime \prime}\left(C_{1}\right) & =-\frac{2(1+b)}{(1-b) e^{i \beta}} \\
& \equiv-\frac{1}{K}
\end{aligned}
$$

From $[1,(1.3)]$ we then have

$$
\frac{\epsilon}{g^{\prime}(u)}=\frac{u}{2}\left\{\frac{\lambda K}{C_{1}^{2}} \frac{C_{1}+u}{C_{1}-u}+\operatorname{con}\left(\frac{\lambda K}{C_{1}^{2}}\right) \frac{1+\bar{C}_{1} u}{1-\bar{C}_{1} u}\right\}+O\left(\lambda^{2}\right) .
$$

To compute $\delta f(z) / g^{\prime}(u)$, we note that from Equation $(4.3) f^{\prime}(z)=g^{\prime}(u) F^{\prime}(z)$ and $f^{\prime \prime}(C)=g^{\prime \prime}\left(C_{1}\right) F^{\prime}(C)^{2}$ since $g^{\prime}\left(C_{1}\right)=0$. Then using Equations (4.6) and $[1,(1.3)]$ we find

$$
\frac{\delta f(z)}{g^{\prime}(u)}=\frac{z F^{\prime}(z)}{2}\left\{\frac{\lambda K}{C^{2} F^{\prime}(C)^{2}} \frac{C+z}{C-z}+\operatorname{con}\left(\frac{\lambda K}{C^{2} F^{\prime}(C)^{2}}\right) \frac{1+\bar{C} z}{1-\bar{C} z}\right\}+O\left(\lambda^{2}\right) .
$$

Equations (4.8), (4.7) and (2.8) now combine to give 


$$
\begin{gathered}
F^{*}(z)=F(z)+\frac{z F^{\prime}(z)}{2}\left\{\frac{\lambda K}{C^{2} F^{\prime}(C)^{2}} \frac{C+z}{C-z}+\operatorname{con}\left(\frac{\lambda K}{C^{2} F^{\prime}(C)^{2}}\right) \frac{1+\bar{C} z}{1-\bar{C} z}\right\} \\
-\frac{F(z)}{2}\left\{\frac{\lambda K}{F(C)^{2}} \frac{F(C)+F(z)}{F(C)-F(z)}+\operatorname{con}\left(\frac{\lambda K}{F(C)^{2}}\right) \frac{1+\bar{F}(C) F(z)}{1-\bar{F}(C) F(z)}\right\}+O\left(\lambda^{2}\right)
\end{gathered}
$$

where $K$ is defined by Equation (4.6) and $F(C)=b^{1 / 2} e^{i \beta}$. Finally we observe that for fixed $F(z)$ and fixed $C, K$ is a nonzero constant, so that $\lambda K \rightarrow 0$ as $K \rightarrow 0$, and $O\left(\lambda^{2}\right)=O\left(K^{2} \lambda^{2}\right)$. This gives the following result.

Theorem 3. Let $F(z) \in U B$. Then for each complex number $C, 0<|C|<1$, and for all complex $\mu,|\mu|<\mu_{0}(C, F)$, there is a function $F^{*}(z) \in U B$ given by Equation (4.9) with $\lambda K$ replaced by $\mu$ and $O\left(\lambda^{2}\right)$ replaced by $O\left(\mu^{2}\right)$.

5. Univalent functions with symmetry. Let $\operatorname{US}(q)$ denote the subclass of $U$ of functions $F(z)$ which are $q$-fold symmetric, that is functions which satisfy the equation $F\left(e_{1} z\right)=e_{1} F(z)$, where $e_{m}=\exp (2 \pi i m / q)$. We again use the method of the preceding paragraphs. In this case we set $g(u)=u-u^{q+1} / C_{1}^{q}(q+1)$, where $C_{1}=F(C), 0<|C|<1$. If $C_{1}$ is subjected to the variation $\lambda$, then each branch point $B_{m}$ of $f(z)=g(F(z))$ is varied by $\lambda e_{m} q /(q+1), m=1,2, \cdots, q$. When the indicated computations are carried out, we arrive at the following theorem.

Theorem 4. Let $F(z) \in \mathrm{US}(q), q$ a positive integer. Then for each complex number $C, 0<|C|<1$, and for all complex $\mu,|\mu|<\mu_{0}(C, F)$, the function

$$
\begin{aligned}
F_{*}(z)= & F(z)\left\{1-2 \Re \frac{\mu}{C^{2} F^{\prime}(C)^{2}}\right\}+z F^{\prime}(z)\left\{\frac{\mu}{C^{2} F^{\prime}(C)^{2}} \frac{C^{q}+z^{q}}{C^{q}-z^{q}}\right. \\
& \left.+\operatorname{con}\left(\frac{\mu}{C^{2} F^{\prime}(C)^{2}}\right) \frac{1+\bar{C}^{q} z^{q}}{1-\bar{C}^{q} z^{q}}\right\}-\frac{2 F(z)^{q+1} \mu}{F^{2}(C)\left(F(C)^{q}-F(z)^{q}\right)}+O\left(\mu^{2}\right)
\end{aligned}
$$

belongs to the class $\mathrm{US}(q)$.

6. A variation formula for multivalent functions. The procedure of $\$ 2$ can also be used to obtain a variation formula for multivalent functions. We will prove

THEOREM 5. Let $F(z)=z+\cdots$ be regular and p-valent in $|z|<1$. Let $b \neq 0$ be a complex number such that $b$ is neither a branch point of the image of $|z|<1$ under $F(z)$, nor a limit value of $F(z)$ as $|z| \rightarrow 1$, and let the equation $F(z)=b$ have $s$ roots, $z_{j}(j=1,2, \cdots, s)$ in $|z|<1$. Then for all complex $\lambda$, $|\lambda|<\lambda_{0}(b, F)$ the function

$$
\begin{aligned}
& F_{\lambda}(z)=F(z)+\frac{\lambda F(z)^{2}}{b(F(z)-b)}-F(z) \sum_{j=1}^{s} \frac{\lambda b}{z_{j}^{2} F^{\prime}\left(z_{j}\right)^{2}} \\
& +z F^{\prime}(z) \sum_{j=1}^{s} \frac{\lambda b}{z_{j} F^{\prime}\left(z_{j}\right)^{2}} \frac{1}{z_{j}-z}+z F^{\prime}(z) \sum_{j=1}^{s} \operatorname{con}\left(\frac{\lambda b}{z_{j} F^{\prime}\left(z_{j}\right)^{2}}\right) \frac{z}{1-\bar{z}_{j} z}+O\left(\lambda^{2}\right)
\end{aligned}
$$


is regular and p-valent in $|z|<1$. Further $F(z)$ and $F_{\lambda}(z)$ have the same number of zeros in $|z|<1$, and have the same number of critical points there.

Proof. Set $g(u)=u-u^{2} / 2 b$, and let

$$
f(z)=g(F(z))=F(z)-\frac{F^{2}(z)}{2 b} .
$$

Clearly $f(z)$ is at most $2 p$-valent in $|z|<1$, and if $R$ denotes the Riemann surface on to which $f(z)$ maps $|z|<1$, then $R$ has first order branch points wherever $R$ lies over the point $B=b / 2=g(b)=f\left(z_{j}\right)$. The surface $R$ may have other branch points, but under the conditions imposed on $b$, these will not lie over $w=b / 2$. We next form $R^{*}$ from $R$ by varying each of the branch points lying over $b / 2$ by the same amount $\lambda$, and let $f^{*}(z)$ be the function mapping $|z|<1$ onto $R^{*}$ preserving the origin and directions there. The Formula (1.3) of [1] is valid when one branch point is altered, but this formula can be applied successively to each of the branch points in question, and it is then clear that

$$
\begin{aligned}
f^{*}(z)=f(z)-\frac{z f^{\prime}(z)}{2}\left\{\sum_{j=1}^{s} \frac{\lambda}{z_{j}^{2} f^{\prime \prime}\left(z_{j}\right)}\right. & \frac{z_{j}+z}{z_{j}-z} \\
& \left.+\sum_{j=1}^{s} \operatorname{con}\left(\frac{\lambda}{z_{j}^{2} f^{\prime \prime}\left(z_{j}\right)}\right) \frac{1+\bar{z}_{j} z}{1-\bar{z}_{j} z}\right\}+O\left(\lambda^{2}\right) .
\end{aligned}
$$

Next let $g^{*}(u)=u-u^{2} /(2 b+4 \lambda)$ and let $G^{*}(w)$ be the inverse function for $g^{*}(u)$. Now $G^{*}(w)$ is not single-valued, but has a simple branch point at $w=B+\lambda$. But since the surface $R^{*}$ has simple branch points at each place which lies over $w=B+\lambda$, the function $G^{*}(w)$ is uniform on $R^{*}$ and therefore $F^{*}(z)=G^{*}\left(f^{*}(z)\right)$ is a single-valued regular function in $|z|<1$. Using Equation (2.8) with $u=F(z), \epsilon=\lambda u^{2} / b^{2}+O\left(\lambda^{2}\right), f^{\prime}(z)=(1-F(z) / b) F^{\prime}(z)$ and $f^{\prime \prime}\left(z_{j}\right)$ $=-F^{\prime}\left(z_{j}\right)^{2} / b$ we find

$$
\begin{aligned}
& F^{*}(z)=F(z)+\frac{\lambda F(z)^{2}}{b(F(z)-b)}+\frac{z F^{\prime}(z)}{2}\left\{\sum_{j=1}^{s} \frac{\lambda b}{z_{j}^{2} F^{\prime}\left(z_{j}\right)^{2}} \frac{z_{j}+z}{z_{j}-z}\right. \\
&\left.+\sum_{j=1}^{s} \operatorname{con}\left(\frac{\lambda b}{z_{j}^{2} F^{\prime}\left(z_{j}\right)^{2}}\right) \frac{1+\bar{z}_{j} z}{1-\bar{z}_{j} z}\right\}+O\left(\lambda^{2}\right) .
\end{aligned}
$$

To normalize, set $F_{*}(z)=F^{*}(z) / F^{*^{\prime}}(0)$. If we use at the same time

$$
\left(z_{j}+z\right) /\left(z_{j}-z\right)=-1+2 z_{j} /\left(z_{j}-z\right)
$$

and

$$
\left(1+\bar{z}_{j} z\right) /\left(1-\bar{z}_{j} z\right)=1+2 \bar{z}_{j} z /\left(1-\bar{z}_{j} z\right)
$$

then Equation (6.4) will give 


$$
\begin{aligned}
F_{*}(z)= & F(z)+\frac{\lambda F(z)^{2}}{b(F(z)-b)}-F(z) \sum_{j=1}^{s} \frac{\lambda b}{z_{j}^{2} F^{\prime}\left(z_{j}\right)^{2}} \\
& +z F^{\prime}(z)\left\{\sum_{j=1}^{s} \frac{\lambda b}{z_{j} F^{\prime}\left(z_{j}\right)^{2}} \frac{1}{z_{j}-z}+\sum_{j=1}^{s} \operatorname{con}\left(\frac{\lambda b}{z_{j} F^{\prime}\left(z_{j}\right)^{2}}\right) \frac{z}{1-\bar{z}_{j} z}\right\} \\
& +i\left(F(z)-z F^{\prime}(z)\right) \Im \sum_{j=1}^{s} \frac{\lambda b}{z_{j}^{2} F^{\prime}\left(z_{j}\right)^{2}}+O\left(\lambda^{2}\right) .
\end{aligned}
$$

Finally set

$$
\begin{aligned}
F_{\lambda}(z) & =e^{i \alpha} F_{*}\left(e^{-i \alpha} z\right)=\left(1+i \alpha+O\left(\alpha^{2}\right)\right)\left(F_{*}(z)-i z F_{*}^{\prime}(z) \alpha+O\left(\alpha^{2}\right)\right) \\
& =F_{*}(z)+i \alpha\left(F_{*}(z)-z F_{*}^{\prime}(z)\right)+O\left(\alpha^{2}\right) .
\end{aligned}
$$

It is clear that this double rotation can always be used to remove from a formula any real multiple of $i\left(F(z)-z F^{\prime}(z)\right)$. If we set $\alpha=-\Im \sum \lambda b / z_{j}^{2} F^{\prime}\left(z_{j}\right)^{2}$ in (6.6) and apply this to $F_{*}(z)$ given by (6.5) we obtain the function defined by Equation (6.1).

To prove that $F_{\lambda}(z)$ has the properties asserted in the theorem it is obviously sufficient to consider $F^{*}(z)$. Let $S$ and $S^{*}$ denote the images of $|z|<1$ under $F(z)$ and $F^{*}(z)$ respectively. Let $\Gamma$ be a circular disk in the $u$ plane containing $u=b$ and $u=b+2 \lambda$. The function $G^{*}(g(u))$, where we take that branch preserving the origin, is regular and univalent outside $\Gamma$ except for the simple pole at infinity. Now $\lambda$ and $\Gamma$ can be taken so small that the boundary points of $S$ have positive distance from $\Gamma$, and in fact if $r<1$ is sufficiently close to one, and if $S(r)$ denotes the image of $r<|z|<1$ under $f(z)$, then $S(r) \cap \Gamma$ is empty. We apply the argument principle to show that $F^{*}(z)$ is $p$-valent. Suppose indeed that the equation $F^{*}(z)=a^{*}$ has $t$ roots in $|z|<1$. Then there is a $\rho, r<\rho<1$ such that $\Delta$ arg $\left(F^{*}\left(\rho e^{i \theta}\right)-a^{*}\right)=2 \pi t$ for $0 \leqq \theta \leqq 2 \pi$. Let $K^{*}$ be the curve $F^{*}\left(\rho e^{i \theta}\right)$ and let $K$ be the curve which goes into $K^{*}$ under $G^{*}(g(u))$. Although $G^{*}(g(u))$ is only defined outside of $\Gamma$, it can be extended as a one-to-one continuous mapping of the $u$ plane onto itself, although this extension is no longer regular. If $u=a$ goes into $a^{*}$ under this extended mapping, then $\Delta$ arg $(u-a)=2 \pi t$ as $u$ describes the curve $K$. But $K$ is the projection of a curve on $S$, so that $F(z)$ assumes the value $a t$ times. Since $F(z)$ is $p$-valent in $|z|<1$, it follows that $t \leqq p$, and hence $F^{*}(z)$ is $p$-valent. The same proof shows that $F(z)$ and $F^{*}(z)$ have the same number of zeros in $|z|<1$. Finally, we mention that except for the points which lie over the disk $\Gamma$, the surface $S^{*}$ is the image of $S$ under $G^{*}(g(u))$, and since this function is univalent outside $\Gamma$, the transformation from $S$ to $S^{*}$ preserves the number of branch points and the order of each branch point.

The Formula (6.1) is quite similar in appearance to the variation formula due to Schiffer [3, Equation (27)] in the special case that $q(z)=z /\left(z-z_{0}\right)$. We will show that except for trivial transformations the formulas are the same, so that our proof of Theorem 5 amounts to a second proof of Schiffer's 
formula. With suitable changes in notation to agree with ours, the Schiffer equation $[3$, Equation (27)] is

$$
\begin{aligned}
F^{*}(z)=F(z) & +\frac{\lambda F(z)}{F(z)-b}+z F^{\prime}(z) \sum_{j=1}^{s} \frac{\lambda b}{z_{j} F^{\prime}\left(z_{j}\right)^{2}} \frac{1}{z_{j}-z} \\
& +z F^{\prime}(z) \sum_{j=1}^{s} \operatorname{con}\left(-\frac{\lambda b}{z_{j} F^{\prime}\left(z_{j}\right)^{2}}\right) \frac{z}{1-\bar{z}_{j} z} \\
& -i z F^{\prime}(z) \Im\left(-\frac{\lambda}{b}+\sum_{j=1}^{s} \frac{\lambda b}{z_{j}^{2} F^{\prime}\left(z_{j}\right)^{2}}\right)+O\left(\lambda^{2}\right)
\end{aligned}
$$

First we normalize by dividing by

$$
F^{* \prime}(0)=1-\Re\left(\frac{\lambda}{b}-\sum_{j=1}^{s} \frac{\lambda b}{z_{j}^{2} F^{\prime}\left(z_{j}\right)^{2}}\right)+O\left(\lambda^{2}\right),
$$

and we find a new $p$-valent function

$$
F_{1}^{*}(z)=F_{\lambda}(z)+i\left(F(z)-z F^{\prime}(z)\right) \Im\left(-\frac{\lambda}{b}+\sum_{j=1}^{s} \frac{\lambda b}{z_{j}^{2} F^{\prime}\left(z_{j}\right)^{2}}\right)
$$

where $F_{\lambda}(z)$ is given by Equation (6.1). But then the double rotation defined in Equation (6.6) will carry $F_{1}^{*}(z)$ into $F_{\lambda}(z)$ if the real multiplier $\alpha$ is selected in the obvious way.

\section{REFERENCES}

1. A. W. Goodman, Variation of the branch points for an analytic function, Trans. Amer. Math. Soc. vol. 89 (1958) pp. 277-284.

2. A. C. Schaeffer and D. C. Spencer, The coefficients of schlicht functions, Duke Math. J. vol. 10 (1943) pp. 611-635.

3. M. Schiffer, Variation of the Green function and theory of the p-valued functions, Amer. J. Math. vol. 65 (1943) pp. 341-360.

The Institute for Advanced Study,

Princeton, N. J.

The University of Kentucky, Lexington, Ky. 